Original Article

\title{
PATTERN OF ANTIBIOTICS UTILIZATION IN NEONATAL SEPTICEMIA: A CROSS-SECTIONAL STUDY FROM RURAL TERTIARY CARE HOSPITAL WESTERN MAHARASHTRA, INDIA
}

\author{
SHINDE A. R. ${ }^{1}$, MOHITE R. V. ${ }^{2}$, SHINDE R. V. ${ }^{3}$ \\ ${ }^{1}$ Department of Pharmacology, Prakash Institute of Medical Sciences and Hospital, Urun-Islampur Maharashtra, India, ${ }^{2}$ Department of \\ Community Medicine, Krishna Institute of Medical Sciences Karad Maharashtra, India, ${ }^{3}$ Department of Microbiology, Krishna Institute of \\ Medical Sciences Karad Maharashtra, India \\ Email: rajsinhmohite124@gmail.com \\ Received: 17 Nov 2016 Revised and Accepted: 09 Jan 2017
}

\begin{abstract}
Objective: To assess the quantification of use of antibiotics and to find out empiric antibiotic regimen practiced for neonatal sepsis in rural tertiary health care centre.

Methods: A hospital, record based cross-sectional study was conducted in Neonatal Intensive Care Unit(NICU) at tertiary care hospital located in western Maharashtra, India. The study was planned during the year 2011-12 among 84 neonates with sepsis. Data were collected by using proforma includes demographic details, antibiotic prescriptions and relevant information.

Results: Among the total 84 neonates, max, $60.71 \%$ had a history of term delivery. The proportion of early and late onset of sepsis was $47.61 \%$ and $52.38 \%$ for which total 18 antibiotics were used of which max, $88.88 \%$ were injectables. Amikacin was used in max, $78.57 \%$ neonates followed by cefotaxime, $45.23 \%$ and ampicillin, $35.71 \%$ in single or combination form respectively. Amikacin was used for max; $929 \mathrm{~d}$ followed by cefotaxime, $523 \mathrm{~d}$ and ampicillin $331 \mathrm{~d}$ respectively. Antibiotics used in single, double and multiple regimens were $19.04 \%, 46.42 \%$ and $34.52 \%$ respectively. Empiric antibiotic regimens practiced were cefotaxim+amikacin and cefotaxim+ampicillin, of which max, $80 \%$ patients were treated with the cefotaxim+amikacin antibiotic regimen. Out of 84 neonates max, $70 \%$ were improved at the time of discharge.
\end{abstract}

Conclusion: Neonatal sepsis was well treated by cefotaxim+amikacin empirical injectable regimen with maximum survival.

Keywords: Antibiotic quantification, Empiric antibiotic regimen, Neonates, Sepsis

(C) 2016 The Authors. Published by Innovare Academic Sciences Pvt Ltd. This is an open access article under the CC BY license (http://creativecommons.org/licenses/by/4. 0/) DOI: http://dx.doi.org/10.22159/ijpps.2017v9i3.16210

\section{INTRODUCTION}

Neonatal sepsis is a clinical syndrome characterized by systemic signs of infection and accompanied by bacteraemia in the first month of life [1]. It is responsible for about $30-50 \%$ of the total neonatal deaths in developing countries and leading causes of neonatal mortality in India [2]. Neonatal sepsis is classified according to the time of onset of the disease as early onset of sepsis (EOS) and late onset of sepsis (LOS). EOS is defined as onset of sepsis in the first three days and is mostly the result of vertical transmission of bacteria from mothers to infants during the intrapartum period. LOS is defined as an infection occurring after 1 $\mathrm{w}$ of life is attributed to horizontal transmission of pathogens acquired post-natal (nosocomial or community) [3].

Organisms causing neonatal sepsis in developing countries are klebsiella, e. coli, pseudomonas, salmonella belonged to the gram-negative bacterial group, whereas staphylococcus aureus, coagulase-negative staphylococci are belonged to the gram-positive bacterial group $[4,5]$. Since sepsis is a systemic inflammatory response to infection, isolation of bacteria from blood is considered the gold standard for the diagnosis of sepsis [6]. However, it takes 24 to $48 \mathrm{~h}$ for culture results. Therefore, non-culturedependent methods need to be found out.

Repeated and prolonged courses of antibiotics exposure have resulted in an increase in the prevalence of hospital acquired, antibiotic resistant organisms such as methicillin-resistant Staphylococcus aureus, vancomycin resistant enterococcus, etc [7]. Similarly, drug toxicity and cost of treatment is another issue raised especially when the patient is in NICU management. Evaluation of antibiotic use is, therefore, important since the prevalence of hospital-acquired antibiotic resistance in organisms is increasing in neonates [8]. The aim of present study was to assess the quantification of use of antibiotics and to find out empiric antibiotic regimen practiced for neonatal sepsis in rural tertiary health care centre.

\section{MATERIALS AND METHODS}

After obtaining permission from the Institutional Ethics Committee; Ref. No. KIMSDU/IEC/09/2011, the study was carried out in the NICU of Krishna Hospital and Medical Research Centre, a tertiary care hospital located in western Maharashtra, India. It was a retrospective, record-based type of epidemiological study. It includes assessment of case records of neonates admitted in paediatric NICU department and diagnosed with sepsis. A total of 84 neonates with a diagnosis of septicemia admitted and treated in NICU during a year of $1^{\text {st }}$ Oct. 2011 to $30^{\text {th }}$ Sept. 2012 were included and their records (case papers) viewed in the study.

\section{Inclusion criteria}

Neonates diagnosed as sepsis by the clinicians and admitted during our study period $1^{\text {st }}$ Oct.2011 to $30^{\text {th }}$ Sept 2012.

\section{Exclusion criteria}

Neonates who were discharged or transferred to other ward or hospital or died within $2 \mathrm{~d}$ of NICU stay were excluded from the study.

\section{Data collection}

The data were collected by investigator in NICU, Department of Pediatrics, according to pre-tested structured proforma from the case records of study subjects on the following variables;

Date of birth, weight at birth, gestational age, sex, diagnosis, outcome, and all antibiotic prescriptions (name, dose, frequency, and route of administration) were retrospectively recorded. Neonates were classified into the age of gestation in weeks at the time of birth. Once neonates have been discharged or transferred to other ward or hospital, no further data was collected. We did not get any data regarding drugs given to the mother. 


\section{Statistical analysis}

Data so collected were entered into MS Excel 2007 and analyzed for descriptive statistics, frequency percentage and presented in the tabular and graphical form.

\section{RESULTS}

Total 84 neonates were enrolled in the study of which max, $60.71 \%$ delivered with full term pregnancy and $69.04 \%$ by normal vaginally.
The Mean (SD) weight (Kg.) and hospital stay (days) of neonates were $2.003 \pm 0.62 \mathrm{~kg}$ and $7.8 \pm 7.24 \mathrm{~d}$ respectively. The proportion of male patients was higher $71.42 \%$ than females (table 1 ).

Clinical presentation with which the neonates were presented to the hospital showed variation; we tabulated those complaints in table 2 Max, 25\% neonates were admitted each for intrauterine growth retardation care (IUGR) and refusal to feed followed by fever $20.23 \%$.

Table 1: Demographic characteristics of neonates

\begin{tabular}{ll}
\hline Study variables & Frequency $(\%)$ \\
\hline Gestational age (weeks) & Preterm $(\leq 37 w \mathrm{ks}): 33(39.28 \%)$ \\
Mode & Full term $(\geq 37 \mathrm{wks}): 51(60.71 \%)$ \\
of delivery & Normal: $58(69.04 \%)$ \\
Weight & Cesarean: $26(30.96 \%)$ \\
$(\mathrm{kg})$ & Minimum: 0.97 \\
& Maximum: 3.40 \\
Hospital stay & mean \pm SD: $2.003 \pm 0.6284$ \\
(days) & Minimum: 1 \\
& Maximum: 38 \\
Gender & mean \pm SD: $7.8928 \pm 7.24$ \\
& Male- $60(71.42 \%)$ \\
\hline
\end{tabular}

Table 2: Clinical presentation of neonates in frequency percentage

\begin{tabular}{ll}
\hline Clinical presentation & Frequency (\%) \\
\hline Intra-uterine growth retardation care (IUGR) & $21(25 \%)$ \\
Refusal to feed & $21(25 \%)$ \\
Fever & $17(20.23 \%)$ \\
Respiratory distress & $7(8.33 \%)$ \\
Yellowish discoloration (skin, sclera and urine) & $8(9.52 \%)$ \\
Convulsion & $4(4.76 \%)$ \\
Vomiting/Abd. distension & $4(4.76 \%)$ \\
Not passing stools & $2(2.38 \%)$ \\
\hline
\end{tabular}

A total of 18 antibiotics were used to treat neonatal sepsis for 84 patients during the study period. Cefotaxime and amikacin were the most commonly prescribed antibiotics $(80 \%)$, followed by cefotaxime and ampicillin. Other antibiotics used and their frequency of use, the total number of antibiotic days and average no of antibiotic use are mentioned in table 3.

Table 3: Profile of antibiotics used in neonatal sepsis

\begin{tabular}{llll}
\hline Name of antibiotic & Frequency (\%) use of antibiotics & Total days of antibiotics & Average antibiotic days per neonate \\
\hline Amikacin & $66(78.57 \%)$ & 929 & 14.07 \\
Cefotaxime & $38(45.23 \%)$ & 523 & 13.76 \\
Ampicillin & $30(35.71 \%)$ & 331 & 11.03 \\
Cefoperazone+sulbactum & $7(8.33 \%)$ & 229 & 32.71 \\
Vancomycin & $16(19.04 \%)$ & 189 & 11.81 \\
Metrondazole & $20(23.80 \%)$ & 156 & 7.8 \\
Ceftriaxone & $12(14.28 \%)$ & 105 & 8.75 \\
Ceftazidime & $10(11.90 \%)$ & 80 & 8 \\
Meropenam & $5(5.95 \%)$ & 76 & 15.2 \\
Ofloxacin & $8(9.52 \%)$ & 57 & 7.12 \\
Tobramycin eye drops & $3(3.57 \%)$ & 12 & 4 \\
Crystalline Penicillin & $3(3.57 \%)$ & 9 & 3 \\
Gentamicin & $5(5.95 \%)$ & 6 & 1.2 \\
Cotrimoxazole & $1(1.90 \%)$ & 5 & 5 \\
Mupirocin & $1(1.90 \%)$ & 5 & 5 \\
Clavulanic acid & $2(2.38 \%)$ & 4 & 2 \\
Linezolid & $1(1.90 \%)$ & 3 & 3 \\
Gatifloxacin eye drops & $1(1.90 \%)$ & 1 & 1 \\
\hline
\end{tabular}

Amikacin is showing highest frequency of use, i.e. $78.57 \%$ in neonatal sepsis followed by cefotaxime, $45.23 \%$ and ampicillin, $35.71 \%$ respectively. Amikacin was used for maximum, $929 \mathrm{~d}$ followed by cefotaxime, $523 \mathrm{~d}$ and ampicillin 331days respectively. Average days of antibiotic used were higher with cefoperazone and sulbactum combination $32.71 \mathrm{~d}$ followed by meropenam $15.2 \mathrm{~d}$ and amikacin $14.07 \mathrm{~d}$. Cefotaxim with amikacin is the commonest antibiotic regimen used for the empirical purpose. The $12.5 \%$ of antibiotics are prescribed in the form of fixed dose combinations (sulfamethoxazole+trimethoprim, cefoperazone+sulbactum). 


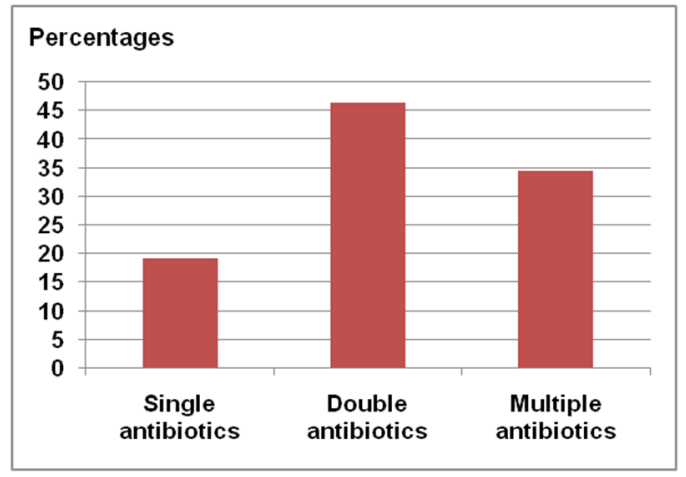

Fig. 1: Antibiotics regimen practiced for treatment of neonatal sepsis

Fig. 1 depicts, the single antibiotic regimen was received by $16(19.04 \%)$ neonates, two antibiotics were received by $39(46.42 \%)$ neonates and more than two antibiotics/multiple antibiotics regimen were received by $29(34.52 \%)$ neonates respectively.

According to the data available on the condition of patients at the time of discharge, they are divided into improved (70\%), expired (25\%) and Discharge against Medical Advice (DAMA) (5\%) as shown in fig. 2.

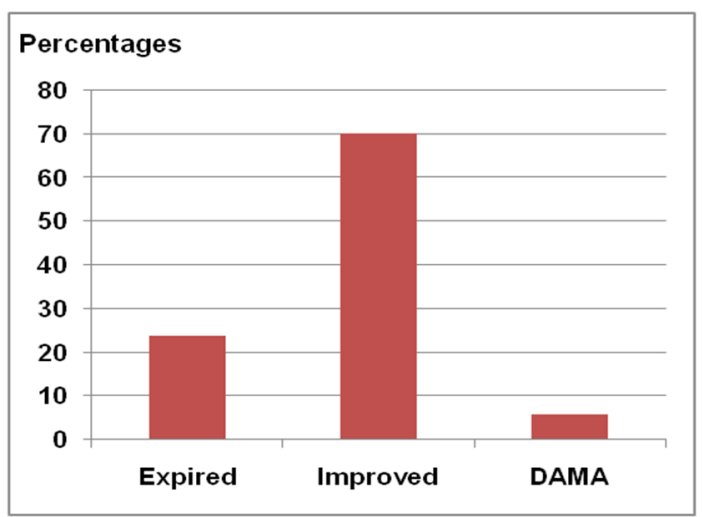

Fig. 2: Treatment outcome of neonatal septicemia

\section{DISCUSSION}

The present study revealed, among total 84 neonates admitted with sepsis max, proportions of neonates were full term $60.71 \%$ and males $71.42 \%$ by gender. A hospital-based study done at Rawalpindi enrolled 104 infants out of which 50\% were full term infants [9]. The differences in results could be due to the quality of ante-natal health care services provided in India. A study conducted in Malaysia included 121 neonates with 58\% males which were less than present study could be due to male preference or gender discrimination in India. Mean birth wt. in our study was $2.003 \pm 0.62$ Kg. whereas, it was $2.75 \pm 0.63 \mathrm{~kg}$ in Malaysian study [10]. This difference in mean birth wt. might be because of the prevalence of maternal malnutrition in rural India.

The study described common presenting complaints were IUGR care (25\%), refusal to feed $(25 \%)$ and fever $(20.43 \%)$ whereas, according to Malaysian study, respiratory distress was main problem (18.2\%) followed by none(12.39\%)and jaundice(10.7\%) [10]. Another study reported respiratory distress $(100 \%)$ followed by abdominal distension $(71.42 \%)$ and signs of pneumonia $(28.57 \%)$ of neonatal sepsis [11]. Unusual presentation of generalised neonatal seizures was seen in a study [12]. These complaints were different than ours. The proportion of early and late onset of sepsis was, $47.61 \%$ and $52.38 \%$ respectively. Total 18 antibiotics were used of which, $88.88 \%$ were injectables. The Amikacin was used in, $78.57 \%$ neonates followed by cefotaxime, $45.23 \%$ and ampicillin, $35.71 \%$ respectively. On an average, cefoperazone with sulbactum was used for $32 \mathrm{~d}$ followed by meropenam $15 \mathrm{~d}$ and amikacin $14 \mathrm{~d}$ respectively. Prolonged duration of antibiotic therapy ( $\geq 5$ days) was associated with necrotising enterocolitis (NEC) or death. Each additional day of antibiotic therapy was associated with $4 \%$ increase in odds of NEC or death [13]. Antibiotic use in the form of single, double and more than double regimen was received by $19.04 \%$, $46.42 \%$ and $34.52 \%$ respectively. Most commonly used an antibiotic in our study was amikacin (78.57\%). It is a drug with very narrow therapeutic range and can cause side effects, such as nephrotoxicity and ototoxicity if 'trough blood level' in excess go uncorrected [14]. The toxicity is increased if amikacin is prescribed for more than $10 \mathrm{~d}$ and when two or more aminoglycosides are prescribed together [15]. In our study, dose interval for amikacin was $12 \mathrm{~h}$ for all neonates, means in a day two doses are needed. In a study, where once vs. multiple daily dosing was studied in Saudi showed that once-daily dosing is preferred over multiple in efficacy, nephrotoxicity and less need of therapeutic drug monitoring [16]. Another study recommended a new dosing regimen, $15 \mathrm{mg} / \mathrm{kg}$ at $36 \mathrm{hr}$ intervals, 14 $\mathrm{mg} / \mathrm{kg}$ at $24 \mathrm{hr}$ intervals for and $15 \mathrm{mg} / \mathrm{kg}$ at $24 \mathrm{hr}$ intervals for neonates $\leq 28$ weeks, $29-36 \mathrm{w}$ and $\geq 37$ weeks postmenstrual age [17]. These findings differ from ours as in our study dosing interval was fixed i.e. $12 \mathrm{H}$ but doses where 20,15 and $10 \mathrm{mg} / \mathrm{kg}$ respectively.

In our study vancomycin was used in $(19.04 \%)$ neonates. Data shows that therapeutic drug monitoring of vancomycin is highly recommended as it is potentially nephrotoxic. It also ensures efficacy and avoids resistance $[18,19]$. A study conducted in Karachi recommended vancomycin+amikacin or carbapenem as the drug of choice for empirical antibiotic regimen treatment of neonatal sepsis [20]. Approximately, $14.28 \%$ low birth weight neonates (Weight $\leq$ $1.5 \mathrm{~kg})$ received third generation Cephalosporins with $39 \%$ mortality in among them. It is reported in previously published studies that use of third generation cephalosporins increases the risk of invasive candidiasis and death in low birth weight neonates (weight $\leq 1.5 \mathrm{~kg}$ ) $[21,22]$. Studies also suggest that third-generation cephalosporins are not more effective in treating sepsis than benzylpenicillin and gentamicin [23]. Previous studies recommended cephalosporins to be used as second line drugs [24]. However in our study cephalosporins were first line drugs whereas benzylpenicillin was not used and gentamicin was used in 5.95\% neonates. The study reported, $70 \%$ neonates with sepsis were improved, and $25 \%$ expired. Malaysian study reported higher improvement rate $88.4 \%$ and mortality $3.3 \%$ [10]. The difference in results could be the use of intrapartum antibiotic prophylaxis, use of antiseptic to disinfect the birth canal, implementation of hand washing, promotion of clean deliveries, well-equipped health care facilities in Malaysian hospitals. Most suspected early onset sepsis neonates with negative blood cultures were given antibiotics, discontinuing empiric antibiotics when blood cultures are negative in neonates can reduce antimicrobial exposure without compromising clinical outcome. Rational prescribing of antibiotics for empirically use should be encouraged so the development of antibiotic resistance can be prevented and cost of treatment will also get minimised [15, 25-27].

\section{CONCLUSION}

Neonatal sepsis would be well managed by Cefotaxim + Amikacin/ Cefotaxime and Ampicillin antibiotic empirical regimen in neonates with birth weight $>1.5 \mathrm{~kg}$. Survival rate would be increased if newborn birth weight increases to normal birth weight. Rational use of antibiotics, avoid development of new drug-resistant strains. Establishment of drug counselling centre to avoid mis-use of any antibiotics. Establishment of hospital infection control committee to avoid hospital acquired infections.

\section{Limitations}

Short duration of study, limited sample size and single study unit

\section{CONFLICT OF INTERESTS}

Declared none

\section{REFERENCES}

1. Patel S, Oshodi A, Prasad P, Delamora P, Larson E, Zaoutis T, et al. Antibiotic use in neonatal intensive care units and 
adherence with centers for disease control and prevention 12 step campaign to prevent antimicrobial resistance. Pediatr Infectious Disease J 2009;28:1047-51.

2. Clark R, Bloom B, Spitzer A, Gerstmann D. Reported medication use in the neonatal intensive care unit: data from a large national data set. Pediatrics 2006;117:1979-87.

3. Baker C, Barrett F. Group B streptococcal infections in infants. The importance of various serotypes. JAMA 1974; 230:1158-60.

4. Karunasekera K, Pathirana D. A preliminary study on neonatal septicemia in a tertiary referral hospital paediatric unit. Ceylon Med J 1999;44:81-6.

5. Karthikeyan G, Premkumar K. Neonatal sepsis: staphylococcus aureus as the predominant pathogen. Indian J Pediatr 2001;68:715-7.

6. Rahman S, Hameed A, Roghani M. Multidrug-resistant neonatal sepsis in Peshawar, Pakistan. Arch Disease Childhood Fetal Neonatal 2002;87:49-52.

7. Bizzarro M, Gallagher P. Antibiotic-resistant organisms in the neonatal intensive care unit. Semin Perinatol 2007;31:26-32.

8. Cotten C, Taylor S, Stoll B, Goldberg R, Hansen N, Sánchez PJ, et al. Prolonged duration of initial empirical antibiotic treatment is associated with increased rates of necrotizing enterocolitis and death for extremely low birth weight infants. Pediatrics 2009; 123:58-66.

9. Khan D, Khan F, Razzaq A. Therapeutic drug monitoring of amikacin in preterm and term infants. Singapore Med J 2009;50:486-9.

10. Sherwin C, Svahn S, Van der Linden A, Broadbent R, Medlicott $\mathrm{N}$, Reith D. Individualised dosing of amikacin in neonates: a pharmacokinetic/pharmacodynamic analysis. Eur J Clin Pharmacol 2009;65:705-13.

11. Alvarez A, Jia Y, Garcia C. Streptococcus bovis bacteremia in neonates in a predominantly hispanic population. Front Pediatr 2015;3:92.

12. Sengupta $M$, Banerjee S, Das N. Early onset neonatal septicaemia caused by pantoea agglomerans. J Clin Diagn Res 2016;5:1-2.

13. Tripathi N, Cotton C, Smith P. Antibiotic use and misuse in the neonatal intensive care unit. Clin Perinatol 2012;39:61-8.

14. Wiley B. Amikacin-drug uses in pregnancy and the first year of life. Neonatal Formulary 6; 2011. p. 40.

15. Jiang J, Chui N, Huang F, Kao H, Hsu C, Hung H, et al. Neonatal sepsis in the neonatal intensive care unit: characteristics of early versus late onset. J Microbial Immunol Infect 2004; 37:301-6.
16. Abdel-Bari A, Mokhtar M, Sabry N, El-Shafi S, Bazan N. Once versus individualized multiple daily dosing of aminoglycosides in critically ill patients. Saudi Pharm 2011;1:9-17.

17. Awaisu A, Syed A, Mohamed I, Saad A. Antimicrobial's utilisation and outcomes of neonatal sepsis among patients admitted to a University Teaching Hospital in Malaysia. Eastern J Med 2007;12:6-14.

18. Jelassi M, Benlmouden A, Lefeuvre S, Mainardi J, Billaud E. Level of evidence For therapeutic drug monitoring of vancomycin. Therapie 2011;66:29-37.

19. Wiley B. Vancomycin-drug uses in pregnancy and the first year of life: neonatal formulary 6 BMJ Books; 2011. p. 264.

20. Mahmood A, Karamat K, Butt T. Neonatal sepsis: high antibiotic resistance of the bacterial pathogens in a neonatal intensive care unit in Karachi. J Pak Med Assoc 2002;52:348-50.

21. Cotten C, McDonald S, Stoll B, Goldberg R, Poole K, Benjamin D. The association of third-generation cephalosporin use and invasive candidiasis in extremely low-birth-weight infants. Pediatrics 2006;118:717-22.

22. Manzoni P, Farina D, Leonessa M, d'Oulx E, Galletto P, Mostert $M$, et al. Risk factors for progression to invasive fungal infection in preterm neonates with fungal colonisation. Pediatrics 2006;118:2359-64.

23. Downie L, Armiento R, Subhi R, Kelly J, Clifford V, Duke T. Community-acquired neonatal and infant sepsis in developing countries: efficacy of WHO's currently recommended antibiotics-systematic review and meta-analysis. Arch Dis Child 2013;98:146-54.

24. Fernando R, Health P, Menson E. Antimicrobial policies in the neonatal units of the United Kingdom and the republic of Ireland. J Antimicrob Chemother 2008;61:743-5.

25. Zakariya B, Bhat V, Harish B, Arun Babu T, Joseph N. Neonatal sepsis in a tertiary care hospital in South India: Bacteriological profile and antibiotic sensitivity pattern. Indian J Pediatr 2011;78:413-7.

26. Dutta S, Reddy R, Sheikh S, Kalra J, Ray P, Narang A. Intrapartum antibiotics and risk factors for early onset sepsis. Arch Dis Child Fetal Neonatal 2010;95:99-103.

27. Bisht R, Alok A, Singh R, Mittal P. Antibiotic resistance-a global issue of concern. Asian J Pharm Clin Res 2009;2:34-9.

\section{How to cite this article}

- $\quad$ Shinde AR, Mohite RV, Shinde RV. Pattern of antibiotics utilization in neonatal septicemia: a cross-sectional study from rural tertiary care hospital western Maharashtra, India. Int J Pharm Pharm Sci 2017;9(3):60-63. 